\title{
A Comparative Study of Some Functional Properties of Lactobacillus and Enterococcus Isolated from Feces of Normo and Hyper-Cholesterolemic Humans
}

\author{
María de Lourdes Reyes-Escogido ${ }^{1 *}$, Claudia Mercedes Gómez-Navarro', Silvia Daniela Ramírez-Ardila², Francisco Martínez-Pérez², \\ Rodolfo Guardado-Mendoza ${ }^{2}$
}

${ }^{1}$ Departamento de Medicina y Nutrición, División de Ciencias de la Salud. Universidad de Guanajuato. Blvd. Puente del Milenio 1001. Fracción Predio de San Carlos. León, Guanajuato, México

${ }^{2}$ Laboratorio de Genómica de Celomados Grupo Microbiología y Genética, Facultad de Ciencias y Grupo de Investigación de Inmunología y Epidemiología Molecular, Facultad de Salud, Universidad Industrial de Santander, Bucaramanga, Santander, Colombia

\begin{abstract}
There have been reports of an association between an imbalance of gastrointestinal microbiota and several kinds of diseases, mainly metabolic diseases. Lactobacillus and Enterococcus are two genera that form part of the native gastrointestinal microbiota. In this work, we isolated, identified and characterized 26 strains of Lactobacillus and 23 strains of Enterococcus from the feces of normo and hypercholesterolemic humans. We compared the behavior exhibited by all strains at acid $\mathrm{pH}$ and in presence of bile salts, their sensitivity to antibiotics, and their ability to hydrolyze bile salts and to reduce cholesterol in vitro. The highest percentage of all isolated and characterized Lactobacillus strains was from the normocolesterolemic group; in the hypercholesterolemic group, most strains belonged to the Enterococcus genus. The Lactobacillus strains showed greater capacity to reduce cholesterol levels; although this capacity has been related to bile salt hydrolase activity, four of the strains we isolated showed no such activity, but still reduced cholesterol. All strains of both genera isolated from normocholesterolemic participants showed a highest rate of cholesterol reduction than those isolated from hypercholesterolemic participants. Furthermore, the Lactobacillus strains showed greater resistance at $\mathrm{pH} 2.0$, while strains of both genera showed similar survival rates at $\mathrm{pH} 3.0$ and in the presence of bile salts after 24 $\mathrm{h}$. These results support the evidence that a microbial imbalance involving the depletion of beneficial bacteria could be detrimental to the health of the host.
\end{abstract}

Keywords: Enterococcus; Gastrointestinal microbiota; Hypocholesterolemic activity; Lactobacillus

\section{Introduction}

The human gastrointestinal tract (GIT) is colonized by a diverse and complex collection of bacterial species; it is estimated that the colon contains around $70 \%$ of all the microorganisms in the human body [1], forming a balanced and dynamic ecosystem that plays an important role in various human body functions. An imbalance in the composition of intestinal bacteria is associated with a variety of diseases such as dysbiosis, colon cancer and hypercholesterolemia, among others [2]. The gastrointestinal tract contains native microorganisms that positively influence health. The majority of these microorganisms belong to the group of lactic acid bacteria (BAL) that includes the bacterial genera Streptococcus, Enterococcus, Leuconostoc, Pediococcus, Lactococcus, Lactobacillus and others within the order of Lactobacillales, such as the genus Bifidobacterium [3]. At the beginning of the $20^{\text {th }}$ century, Ely Metchnikoff proposed various theories about the positive health effects of the consumption of Lactobacillus; these theories are still accepted and have contributed to the conviction that these bacteria perform a very important function. A study by Marteau et al. 2001 reported that Lactobacillus-Enterococcus comprise $6.6 \%$ of the bacterial population in feces [4]. It has also been reported that Enterococcus, which has been part of gut microbiomes since at least the early Devonian period, comprises a little less than $1 \%$ of the human gastrointestinal microbiota [5]. Although various strains of this genus have been associated with infections, others have been found to have beneficial health effects on the host, and have even been used as probiotics [6]; however, the probiotic effects of this genus have been little studied compared to Lactobacillus. In vivo and in vitro studies of various strains of both genera have shown their potential to reduce cholesterol, alone or in combination with other strains [7]. Various mechanisms have been proposed to explain this beneficial activity, all of them associated with the metabolism of the bacteria, such as hydrolase secretion, which has been related to the removal of cholesterol through the hydrolysis of amide bonds and the releases of bile salts [8], since poorly water soluble bile salts precipitate at low $\mathrm{pH}$, and this is related to the co-precipitation of cholesterol; however, not all the cholesterol precipitates, and a part of it may remain bound to the surface of the bacterial cell [9]. Other mechanisms involve the conversion of cholesterol to coprostanol in the intestine, which is later excreted in the feces, reducing the quantity of cholesterol available to be absorbed by the intestine [10]. Cholesterol can also be used as a substrate for the synthesis of new bile acids in a homeostatic response, resulting in the reduction of steric cholesterol [11]. However, most of the information about these mechanisms corresponds to strains of Lactobacillus and Bifidobacterium, while there is little information regarding the cholesterol reducing capacity of Enterococcus strains and the mechanisms involved [12,13]. It is important to study the behavior of strains native to the gastrointestinal tract in order to establish preventive strategies for hyper-cholesterolemia

*Corresponding author: María de Lourdes Reyes-Escogido, Departamento de Medicina y Nutrición, División de Ciencias de la Salud. Universidad de Guanajuato. Blvd. Puente del Milenio 1001. Fracción Predio de San Carlos. León, Guanajuato, CP. 37670, México., Tel: (+52) 477-2674900 ext. 3683; E-mail: ml.reyes@ugto.mx

Received March 21, 2016; Accepted May 06, 2016; Published May 10, 2016

Citation: Reyes-Escogido ML, Gómez-Navarro CM, Ramírez-Ardila SD, MartínezPérez F, Guardado-Mendoza R (2016) A Comparative Study of Some Functional Properties of Lactobacillus and Enterococcus Isolated from Feces of Normo and Hyper-Cholesterolemic Humans. J Bacteriol Parasitol 7: 274. doi: 10.4172/21559597.1000274

Copyright: (C) 2016 Reyes-Escogido ML, et al. This is an open-access article distributed under the terms of the Creative Commons Attribution License, which permits unrestricted use, distribution, and reproduction in any medium, provided the original author and source are credited. 
Citation: Reyes-Escogido ML, Gómez-Navarro CM, Ramírez-Ardila SD, Martínez-Pérez F, Guardado-Mendoza R (2016) A Comparative Study of Some Functional Properties of Lactobacillus and Enterococcus Isolated from Feces of Normo and Hyper-Cholesterolemic Humans. $\mathrm{J}$ Bacteriol Parasitol 7: 274. doi: 10.4172/2155-9597.1000274

and associated diseases. Statins are generally the most effective drug for controlling high cholesterol levels and preventing disorders related to atherosclerosis [14]; however, although recent studies have shown an increase in the number of patients who consume statins, the number of cases of intolerance or lack of response to statins has also increased [15]. Moreover, statins have been recently considered as a risk factor in the development of liver cancer [16]. The high cost of the drugs, as well as the little willingness to follow a diet and exercise plan, are limiting factors for the effectiveness of pharmacological therapy, which is why the manipulation of the intestinal microbiota has been considered as a good alternative for cholesterol control. It is therefore necessary to study the functional capacity of the native microbiota and its potential contribution to human health.

\section{Material and Methods}

\section{Participants}

A total of 65 adult volunteers participated in the study; the age range was 18-58 years. We determined the general characteristics of the participants, such as age, sex, body mass index, physical activity, and nutrient consumption; we also determined their glucose and blood cholesterol levels. The participants were asked to avoid consuming fermented foods for at least one week prior to the collection of the fecal sample and isolation of bacteria; they were also asked not to take any antibiotics or anti-inflammatory drugs for one month prior to the study, and to confirm that they did not suffer from any gastrointestinal condition. This study was conducted following the Guidelines for Good Clinical Practice and the Helsinki declaration; informed consent was obtained from all individual participants included in the study.

\section{Nutritional and physical activity assessment}

A previously validated semi-quantitative food frequency questionnaire (FFQ) was used to evaluate dietary intake. This questionnaire included data regarding the consumption of 116 food items. A commonly used portion size was specified for each food (e.g. 1 slice of bread or 1 cup of coffee) in the FFQ and the participants reported their frequency of consumption of each food over the previous year. The participants chose from 10 possible responses, ranging from "never" to "6 or more times per day." For our analysis, the reported frequency for each food item was converted into daily intake values. Total energy intake was computed by summing the energy intake from all foods. The PA level of the participants was assessed using a validated Spanish version of a self-administered questionnaire [17] adapted for use in the Mexican population.

\section{Isolation of lactic acid bacteria}

Serial dilution techniques were used to isolate the bacteria. Each sample suspension was prepared by adding $1 \mathrm{~g}$ of feces to $9 \mathrm{ml}$ of peptone water; the fecal matter was then suspended by vigorous stirring in a vortex for at least $1 \mathrm{~min}$. Serial dilutions were conducted to obtain concentrations of $10^{-4}$ and $10^{-5}$, which were spread on MRS agar plates; the plates were then incubated at $37^{\circ} \mathrm{C}$ for $48-72 \mathrm{~h}$ in anaerobiosis. After the growth of microorganisms, pure cultures of bacteria were subcultured in MRS agar and incubated at $37^{\circ} \mathrm{C}$ to promote vigorous growth. We observed the appearance of colonial growth, the reaction to the Gram stain and the microscopic cellular morphology; we confirmed the absence of spores by staining with malachite and through the use of standard biochemical tests (oxidase and catalase).

\section{Susceptibility to antibiotics}

We used the disc diffusion method to evaluate the behavior of the studied strains in the presence of the following antibiotics: Ampicillin $(10 \mu \mathrm{g})$, Gentamicin $(10 \mu \mathrm{g})$, Kanamycin $(30 \mu \mathrm{g})$, Erythromycin $(15$ $\mu \mathrm{g})$, Clindamycin $(2 \mu \mathrm{g})$, Tetracycline $(30 \mu \mathrm{g})$, Chloramphenicol $(30$ $\mu \mathrm{g})$, Streptomycin $(10 \mu \mathrm{g})$, and Neomycin $(30 \mu \mathrm{g})$. The concentrations of each antibiotic were determined according to the guidelines of the Clinical and Laboratory Standards Institute [18]. The turbidity of cultures after $24 \mathrm{~h}$ of incubation was adjusted to obtain a concentration of $1.5 \times 10^{8}$ UFC $\mathrm{ml}^{-1}$ on the McFarland scale. After adjusting the concentrations, the MRS agar plates were inoculated with the bacteria and the antibiotic discs were placed on each plate. After $20 \mathrm{~h}$ of incubation, the halos of inhibition were measured and reported in $\mathrm{mm}$. The results were interpreted as Sensitive (S), Intermediate Sensitivity (I) or Resistant (R) [18].

\section{Tolerance to acid $\mathrm{pH}$ and bile salts $(0.3 \% \mathrm{w} / \mathrm{v})$}

Three different types of MRS broth were prepared; two with $6 \mathrm{M}$ $\mathrm{HCl}$, one adjusted at $\mathrm{pH} 2.0$ and the other at $\mathrm{pH} 3.0$; the third had MRS and bile salts $(0.3 \% \mathrm{w} / \mathrm{v})$ and was adjusted at $\mathrm{pH} 6.5$. Fresh cultures of each strain were used to inoculate each of the three broths mentioned above, using normal MRS broth as control. The inoculated media were incubated at $37^{\circ} \mathrm{C}$ in anaerobiosis; aliquots were taken at specific time intervals to inoculate MRS agar plates. From cultures at $\mathrm{pH} 2.0$, aliquots were taken at $0,0.5,1.5,3$, and $24 \mathrm{~h}$; from the cultures at $\mathrm{pH} 3.0$ and cultures with bile salts (3.0\%), aliquots were taken at 0,3 , and $24 \mathrm{~h}$. All control strains were inoculated at the same time. The inoculated plates were incubated at $37^{\circ} \mathrm{C}$ for $24 \mathrm{~h}$ in anaerobiosis. The survival rate in each type of broth was calculated as the base 10 logarithm of UFC ml-1 and reported as percentage [19].

\section{Hydrolase activity (Bile salt hydrolysis)}

We determined the bile salt hydrolase activity (BSH) of the strains using MRS agar supplemented with sodium thioglycolate $(0.2 \%)$ and taurocholic acid $(0.2 \%)$. To do this, assay filter paper discs $(6 \mathrm{~mm}$ in diameter) were inoculated with fresh culture of the corresponding strains. The disks were placed on the MRS agar plates supplemented with bile salts; the plates were then incubated at $37^{\circ} \mathrm{C}$ for $20-24 \mathrm{~h}$ in anaerobiosis. Hydrolase activity was reported as positive when a precipitate was observed in the middle and around the inoculated discs. The precipitate was cholic acid generated by the enzyme.

\section{In vitro cholesterol reduction}

We prepared MRS broth supplemented with cholesterol at a final concentration of $100 \mu \mathrm{g} \mathrm{ml}^{-1}$. This medium was inoculated with $1 \%$ of fresh culture of each strain; the inoculums were incubated at $37^{\circ} \mathrm{C}$ for $20 \mathrm{~h}$ in anaerobiosis. Uninoculated sterile broth was also analyzed as negative control. After the incubation period, the cells were removed from the medium by centrifugation $(10,000 \times \mathrm{g}$ for $15 \mathrm{~min})$, and the supernatant was recovered to determine residual cholesterol using a colorimetric method. The percentage of cholesterol reduction was calculated using the following formula:

$$
\% \text { Reduction }=100-\frac{B}{C} \cdot 100
$$

Where, B: Absorbance of MRS-cholesterol from removed cells; C: Absorbance of MRS-cholesterol from uninoculated broth.

\section{Molecular identification}

The extraction of genomic DNA was performed from pure cultures after $24 \mathrm{~h}$ of incubation, using a commercial kit (Purelink genomic DNA Kit, Invitrogen, CA, USA). The extracted DNA was used as a template for the partial amplification of the 16S rRNA gene using the 
Citation: Reyes-Escogido ML, Gómez-Navarro CM, Ramírez-Ardila SD, Martínez-Pérez F, Guardado-Mendoza R (2016) A Comparative Study of Some Functional Properties of Lactobacillus and Enterococcus Isolated from Feces of Normo and Hyper-Cholesterolemic Humans. $\mathrm{J}$ Bacteriol Parasitol 7: 274. doi: 10.4172/2155-9597.1000274

primers forward 5'-AGAGTTTGATCCTGGCTCAG-3' and reverse 5'-AAGGAGGTGATCCAGCCGCA-3' [20]. The PCR products were visualized on agarose gels stained with Ethidium bromide $(0.5 \mu \mathrm{g} \mathrm{ml}$ ${ }^{1}$ ). The PCR products were purified using a commercial kit (PureLink PCR purification kit, Invitrogen, CA, USA) and then sent to the IPICYT, A.C. San Luis Potosi, Mexico, for sequencing. The sequence analysis was carried out using the Basic Local Alignment Search Tools (BLAST) from the database of the National Center for Biotechnology Information (NCBI).

\section{Statistical analysis}

All experiments were performed in triplicate and the results are shown as mean $\pm S D$; $<<0.05$ was considered statistically significant.

\section{Results and Discussion}

The 65 participants had normal glucose levels; of them, 25 had high cholesterol levels ( $\geq 200 \mathrm{mg} \mathrm{dl}^{-1}=$ hypercholesterolemic group, $\mathrm{HC}$ ) and 40 had normal cholesterol levels $\left(<200 \mathrm{mg} \mathrm{dl}^{-1}=\right.$ normocholesterolemic group, NC). As is known, the intestinal microbiota seems to have a clear role in human health [3], and the diet is considered an important factor in its modulation; we didn't find any significant difference in the nutritional and physical activity profile between the study groups. The body mass index (BMI) tended to be higher in the HC group, in which $84 \%$ of the participants were overweight or obese, and more than $50 \%$ had obesity; in the NC group, $25 \%$ had obesity, $40 \%$ were overweight, and $35 \%$ had normal weight. As can be seen in Table 1, the main differences between groups were determined by the participants with normal weight or obesity. Regarding physical activity, the NC group showed higher physical activity than the HC group (Table 1). Several studies have reported that physical activity, obesity, and diet influence the composition and function of the microbiota [21-23], however, there is still not enough knowledge about the relationship between these factors and the composition and function of microbiota [24]. In this study, we did not find any differences between groups regarding the consumption of nutrients, although the tendency of the NC group to perform more physical activity implies an increased demand of energy. The intestinal microbiota is involved in several activities, such as energy production, synthesis of micronutrients, fermentation processes and absorption of electrolytes, which regulates both nutrient acquisition and energy extraction through the synthesis of enzymes involved in the metabolism of carbohydrates, cholesterol and proteins [25]. As it was reported in a previous study in rats, both the amount of food consumed and physical activity influence the function and composition of the native microbiota, affecting the host's health [26].

Of all the isolated strains, 247 were identified as non-sporulating bacteria, Gram-positive and negative to catalase and oxidase tests [27]. We determined the sensitivity of the 247 strains to nine antibiotics, setting a threshold for elimination of resistance to 3 or more antibiotics; only the $37.6 \%$ of the strains were selected, being sensitive to at least seven antibiotics. The selected strains were identified by partial sequencing of the $16 \mathrm{~S}$ rRNA ribosomal gene, but only 67 of these sequences were deposited in the Genbank database after several duplicate sequences were identified. Once identified, 49 strains of the Lactobacillus and Enterococcus genera (Table 2) were selected. Twentysix strains of the genus Lactobacillus and 23 of the genus Enterococcus were evaluated, comparing their behavior under different conditions. Seventy-six point nine percent of the Lactobacillus strains were isolated from the NC group, while $52.2 \%$ of the Enterococcus strains were isolated from the $\mathrm{HC}$ group; $\mathrm{p}=0.03$ (Figure 1). The ratio between Lactobacillus and Enterococcus was 1.6 in the NC group and 0.4 in the HC group. Some studies have reported that the proportion of these two genera changes with the presence of Inflammatory Intestinal Disease; these changes were reflected in the depletion of Lactobacillus and the increase of Enterococcus [28], as we observed in our study, although our participants have hypercholesterolemia, the oxidative stress is the common factor in both cases. In this study, we observed that the

\begin{tabular}{|c|c|c|c|c|}
\hline & $N C(n=40)$ & $\mathrm{HC}(\mathrm{n}=25)$ & $p$ & \\
\hline Age (years) & $34.05 \pm 13.40$ & $42.40 \pm 10.76$ & 0.008 & * \\
\hline \multicolumn{5}{|l|}{ Gender \% } \\
\hline Female, n (\%) & $(55.00)$ & $(60.00)$ & \multirow{2}{*}{0.692} & \multirow{2}{*}{ ** } \\
\hline Male, n (\%) & $(45.00)$ & $(40.00)$ & & \\
\hline BMI $\left(\mathbf{k g} / \mathbf{m}^{2}\right)$ & $27.67 \pm 5.90$ & $29.44 \pm 4.15$ & 0.195 & * \\
\hline Normal weight & 35.00 & 16.00 & \multirow{3}{*}{0.066} & \multirow{3}{*}{ ** } \\
\hline Overweight & 40.00 & 32.00 & & \\
\hline Obesity & 25.00 & 52.00 & & \\
\hline \multicolumn{5}{|l|}{ Physical activity } \\
\hline Low & 35.00 & 40.00 & \multirow{3}{*}{0.902} & \multirow{3}{*}{ ** } \\
\hline Moderate & 32.50 & 32.00 & & \\
\hline High & 32.50 & 28.00 & & \\
\hline \multicolumn{5}{|l|}{ Biochemicals } \\
\hline Glucose (mg/dl) & $93.30 \pm 25.49$ & $94.92 \pm 19.49$ & 0.787 & * \\
\hline TC (mg/dl) & $163.86 \pm 27.72$ & $228.48 \pm 23.46$ & 0.000 & * \\
\hline \multicolumn{5}{|l|}{ Dietary assessment } \\
\hline Energy (kcal/d) ${ }^{a}$ & $2455.21 \pm 1111.52$ & $2398.70 \pm 930.29$ & 0.832 & *** \\
\hline Carbohydrates $(g / d)^{a b}$ & $318.36 \pm 162.13$ & $309.10 \pm 160.69$ & 0.592 & *** \\
\hline Protein $(g / d) b$ ab & $83.36 \pm 43.60$ & $79.59 \pm 21.00$ & 0.458 & $* * *$ \\
\hline Lipids $(g / d)^{a b}$ & $45.47 \pm 25.06$ & $39.09 \pm 15.66$ & 0.171 & *** \\
\hline Cholesterol (mg/d) ab & $283.23 \pm 176.55$ & $273.79 \pm 154.36$ & 0.797 & $* * *$ \\
\hline
\end{tabular}

BMI: Body Mass Index, TC: total cholesterol. ${ }^{*}$ Results from a Student's $t$ test and ${ }^{* *}$ Chi-square. Results presented as mean \pm standard deviation and percentage. ${ }^{* * *}$ General linear model adjusted. for ${ }^{a}$ age, gender and ${ }^{b}$ energy; Data are presented as mean \pm standard deviation.

Table 1: General characteristic of the study groups. 
Citation: Reyes-Escogido ML, Gómez-Navarro CM, Ramírez-Ardila SD, Martínez-Pérez F, Guardado-Mendoza R (2016) A Comparative Study of Some Functional Properties of Lactobacillus and Enterococcus Isolated from Feces of Normo and Hyper-Cholesterolemic Humans. $\mathrm{J}$ Bacteriol Parasitol 7: 274. doi: 10.4172/2155-9597.1000274

\begin{tabular}{|c|c|c|}
\hline Identified strain & $\begin{array}{c}\text { Genbank } \\
\text { access }\end{array}$ & Origin \\
\hline Lactobacillus $s p$ strain L101(LBF2)E04 & KM269715 & NC \\
\hline Lactobacillus sp strain L112(LBF2)F03 & KM269716 & NC \\
\hline Lactobacillus $s p$ strain L238(LBF2)G05 & KM269717 & NC \\
\hline Lactobacillus sp strain L314(LBF2)H05 & KM269718 & NC \\
\hline Lactobacillus $s p$ strain L319(LBF2)C02 & KM269719 & NC \\
\hline Lactobacillus $s p$ strain L329(LBF2)D02 & KM269720 & NC \\
\hline Lactobacillus plantarum strainA05_0330_01 & KP340446 & NC \\
\hline Lactobacillus fermentum strain L634(LBF2)G03 & KM269711 & NC \\
\hline Lactobacillus sp strain L656(LBF2)H03 & KM269710 & $\mathrm{HC}$ \\
\hline Lactobacillus sp strain L729(LBF2)C04 & KM269721 & NC \\
\hline Lactobacillus plantarum strain 793_E01 & KP178096 & NC \\
\hline Lactobacillus plantarum strain 820_H01 & KP178097 & $\mathrm{HC}$ \\
\hline Lactobacillus plantarum strain 821_A01 & KP178098 & $\mathrm{HC}$ \\
\hline Lactobacillus plantarum strain D05_0854_04 & KP340447 & $\mathrm{HC}$ \\
\hline Lactobacillus plantarum strain 857_C02 & KP178099 & $\mathrm{HC}$ \\
\hline Lactobacillus plantarum strain 904_D02 & KP178100 & NC \\
\hline Lactobacillus $s p$ strain 906_E02 & KP178101 & NC \\
\hline Lactobacillus casei strain 1070_A04 & KP178092 & NC \\
\hline Lactobacillus sp strain 1265(LBF2)A03 & KM269704 & NC \\
\hline Lactobacillus $s p$ strain 1266(BF2)B03 & KM269705 & NC \\
\hline Lactobacillus $s p$ strain 1280(LBF2)G02 & KM269706 & $\mathrm{HC}$ \\
\hline Lactobacillus ruminis strain 1291(LBF2)H05 & KM269714 & NC \\
\hline Lactobacillus ruminis strain 1292_G05 & KP178094 & NC \\
\hline Lactobacillus sp strain 1311_H05 & KP178109 & NC \\
\hline Lactobacillus ruminis strain 1313_A06 & KP178095 & NC \\
\hline Lactobacillus plantarum strain B08_1534_02 & KP340448 & NC \\
\hline Enterococcus faecium strain L030(LBF2)D03 & KM269699 & NC \\
\hline Enterococcus durans strain L106(LBF2)E03 & KM269697 & $\mathrm{HC}$ \\
\hline Enterococcus faecium strain L175(LBF2)F04 & KM269700 & $\mathrm{HC}$ \\
\hline Enterococcus faecium strain L179(LBF2)G04 & KM269701 & $\mathrm{HC}$ \\
\hline Enterococcus faecium strain L180(LBF2)H04 & KM269702 & $\mathrm{HC}$ \\
\hline Enterococcus faecalis strain B04_0182_02 & KP340440 & $\mathrm{HC}$ \\
\hline Enterococcus durans strain L186(LBF2)A05 & KM269698 & $\mathrm{HC}$ \\
\hline Enterococcus sp strain C04_0189_03 & KP340441 & NC \\
\hline Enterococcus hirae strain L217(LBF2)C05 & KM269707 & $\mathrm{HC}$ \\
\hline Enterococcus hirae strain L220(LBF2)D05 & KM269708 & $\mathrm{HC}$ \\
\hline Enterococcus hirae strain L222(LBF2)E05 & KM269709 & $\mathrm{HC}$ \\
\hline Enterococcus faecium strain 344(F)_E02 & KP178084 & NC \\
\hline Enterococcus sp strain 347_D01 & KP178087 & NC \\
\hline Enterococcus faecium strain L703(LBF2)A04 & KM269703 & $\mathrm{HC}$ \\
\hline Enterococcus durans strain 794_F01 & KP178088 & NC \\
\hline Enterococcus faecium strain 855_E03 & KP178089 & $\mathrm{HC}$ \\
\hline Enterococcus $s p$ strain 919_B01 & KP178108 & NC \\
\hline Enterococcus sp strain 1284_F05 & KP178106 & $\mathrm{HC}$ \\
\hline Enterococcus hirae strain C07_1387_03 & KP340442 & NC \\
\hline Enterococcus faecium strain 1479_C05 & KP178085 & NC \\
\hline Enterococcus durans strain 1520_D05 & KP178086 & NC \\
\hline Enterococcus faecium strain A09_1535_01 & KP340439 & NC \\
\hline Enterococcus sp strain 1547_E05 & KP178107 & NC \\
\hline
\end{tabular}

Table 2: Strains identified by $16 \mathrm{~S}$ rRNA sequencing, Genbank access and origin.

participants with obesity and high cholesterol levels had a reduced population of beneficial Lactobacillus. The Lactobacillus genus has been identified as one of the most beneficial to human health [29]; it has the highest number of strains with probiotic properties. Although some strains of the genus Enterococcus have been associated with infectious processes [30], others have shown probiotic properties [29]. Given the functional importance of both genera, we evaluated their behavior in different conditions in order to further our knowledge about the

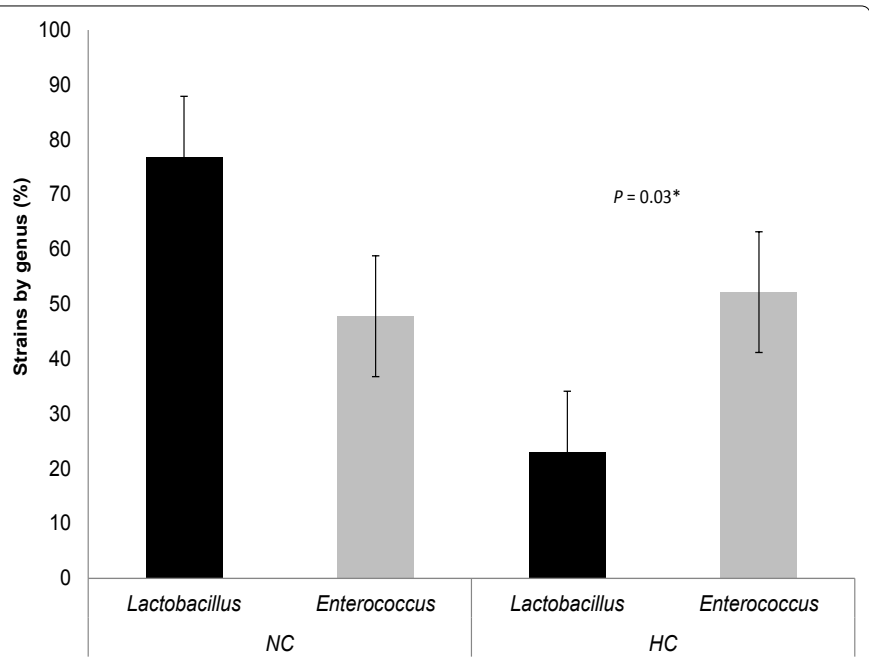

Figure 1: Distribution of Lactobacillus and Enterococcus by origin. NC normocholesterolemic group, $\mathrm{HC}$; hypercholesterolemic group. Black bars, Lactobacillus, gray bars, Enterococcus.

beneficial functions of the native microbiota. An important function of the native microbiota is its contribution to cholesterol metabolism, mainly of exogenous cholesterol (diet); importantly, we did not find any significant differences in the consumption of cholesterol or any other kind of lipids between the NC and HC groups, which reinforce the idea that the observed differences in the microbiota between the study groups was not due to the consumption of cholesterol or lipids. We observed that Lactobacillus has a greater capacity to reduce cholesterol; while Enterococcus can also reduce cholesterol, it has a lower activity than Lactobacillus. However, the cholesterol-reducing ability of the Lactobacillus strains isolated from the HC group was lower than the ability of Lactobacillus strains isolated from the NC group. Similar changes in the proportions of Lactobacillus and Enterococcus strains were reported in a study conducted in rats subjected to changes in diet and physical activity; in that study, the population of Lactobacillus was higher in rats that performed physical activity and were fed an ad libitum diet, while the population of Enterococcus was higher in rats without physical activity and with induced anorexia [26]. One of the proposed mechanisms to reduce cholesterol involves bile salt hydrolase (BSH), an enzyme produced by bacteria. According to our results, with the exception of four strains, all strains showed hydrolase activity; however, all strains reduced cholesterol after $20 \mathrm{~h}$, independently from the presence of this enzyme, including those strains that did not show hydrolase activity, which means that the mechanism by which these strains reduce cholesterol may not be related only to the enzyme. At the genus level, the average cholesterol reduction rate was $56 \%$ for Lactobacillus and 51.3\% for Enterococcus. Analyzing the behavior of the strains according to their origin some differences were observed; the cholesterol reduction rates of Lactobacillus (60.5\%) and Enterococcus (58.2\%) strains isolated from the NC group were higher than the rates observed in the strains isolated from the HC group, which were $50.4 \%$ and $44.9 \%$, respectively. This shows that Lactobacillus is more efficient than Enterococcus in cholesterol reduction. Thus, the restoration of the microbiota could be an alternative for the control of total cholesterol levels and other related lipids such as triglycerides, LDL, VLDL, and HDL, independently of the mechanism used [29].

Several studies have reported that Lactobacillus and Enterococcus strains present resistance to one or more antibiotics, which may be 
Citation: Reyes-Escogido ML, Gómez-Navarro CM, Ramírez-Ardila SD, Martínez-Pérez F, Guardado-Mendoza R (2016) A Comparative Study of Some Functional Properties of Lactobacillus and Enterococcus Isolated from Feces of Normo and Hyper-Cholesterolemic Humans. $\mathrm{J}$ Bacteriol Parasitol 7: 274. doi: 10.4172/2155-9597.1000274

Page 5 of 6

intrinsic or acquired. Lactobacilli have intrinsic resistance to a wide range of antibiotics; however, in the majority of cases, this resistance is non-transferable [31]. These genera are usually sensitive to penicillin, which inhibits the synthesis of the cell wall, as well as to protein synthesis inhibitors such as chloramphenicol, tetracycline and clindamycin [32], which is consistent with the results obtained in this study (Figure 2), in which the sensitivity to penicillin, chloramphenicol and tetracycline was close to $100 \%$, in the case of clindamycin; the $84.6 \%$ of the Lactobacillus strains were identified as sensitive and the $15.4 \%$ was identified as having intermediate sensitivity, and $83 \%$ of Enterococcus strains were sensitive and $13 \%$ were resistant. It has been reported that this genus has an intrinsic resistance to this antibiotic; it could even be considered as a low resistance rate [33]. The sensitivity of Lactobacillus strains to Gentamicin was $92.3 \%$, which was also in accordance with previous reports [34], while for Enterococcus it was 69.6\%, with some strains showing intermediate sensitivity and resistance [33]. In general, the behavior exhibited by the strains in the presence of most antibiotics agrees with that reported in previous studies [35]. The behavior of both genera in the presence of Neomycin was interesting; as can be seen in Figure 3, 3.8\% of the Lactobacillus strains were resistant to this antibiotic, while the resistance of Enterococcus was $8.7 \%$. These rates

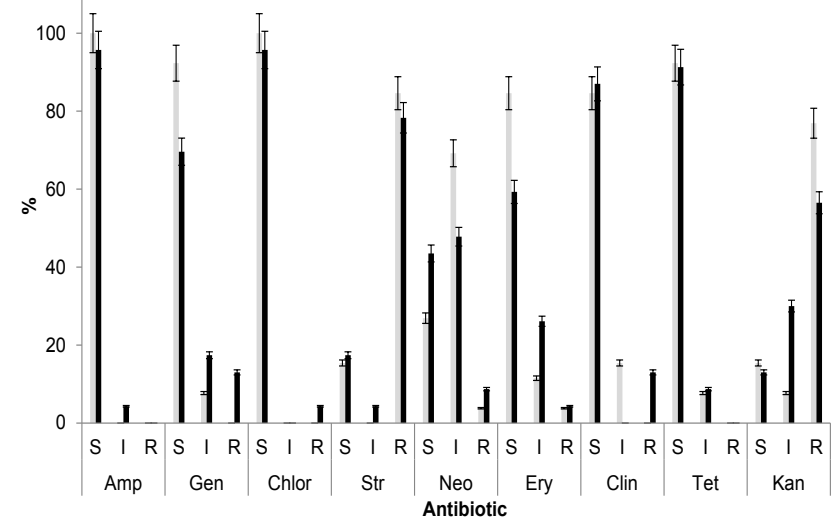

Figure 2: Rates of antibiotic susceptibility of Lactobacillus (gray bars) and Enterococcus (black bars). $\mathrm{S}=$ Sensitive; I= Intermediate sensitivity; $\mathrm{R}=$ Resistant. Amp $=$ Ampicillin, Gen= Gentamicin, Chlo $=$ Chloramphenicol, Str $=$ Streptomycin, Neo= Neomycin, Ery= Erythromycin, Clin= Clindamycin, Tet= Tetracycline, Kan= Kanamycin.

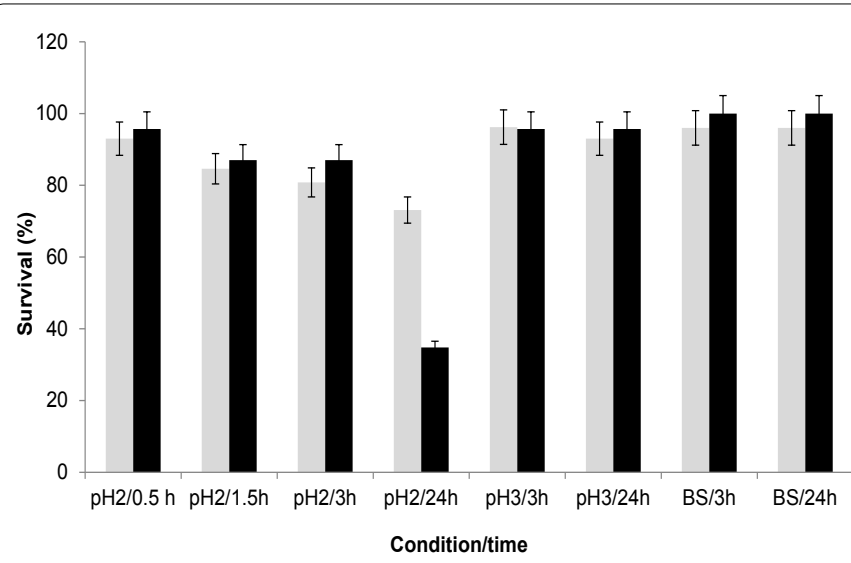

$\mathrm{pH} 2 / 3 \mathrm{~h} \quad \mathrm{pH} 2 / 24 \mathrm{~h} \quad \mathrm{pH} 3 / 3 \mathrm{~h} \quad \mathrm{BS} / 3 \mathrm{~h} \quad \mathrm{BS} / 24 \mathrm{~h}$

Figure 3: Survival rates of Lactobacillus (gray bars) and Enterococcus (black bars) under three different conditions $(\mathrm{pH} 2.0, \mathrm{pH} 3.0,0.3 \%$ bile salts) and times. of resistance are relatively low, since high rates of resistance have been previously reported for Lactobacillus [36] and Enterococcus [37], and these genera have and intrinsic resistance to aminoglycosides, to which neomycin belongs. Although in our results the resistance rates were low, there were also a high percentage of intermediate sensitivity strains in both genera, which may tend towards resistance due to the nature of the bacteria. Finally, when evaluating the survival of the strains at acid $\mathrm{pH}(2.0$ and 3.0), and in the presence of bile salts $(0.3 \% \mathrm{w} / \mathrm{v})$, after $3 \mathrm{~h}$ in the medium adjusted to $\mathrm{pH} 2.0$, Lactobacillus showed a survival rate of $80.8 \%$ and Enterococcus $87.0 \%$; interestingly, after $24 \mathrm{~h}$ under the same conditions, $73.1 \%$ of the Lactobacillus strains and $34.8 \%$ of the Enterococcus strains remained viable; it was under these conditions and after $24 \mathrm{~h}$ that we found a difference in survival between the genera. At $\mathrm{pH}$ 3.0, the survival of Lactobacillus (93\%) and Enterococcus (95.7\%) strains after $24 \mathrm{~h}$ was very similar, as can be seen, while after $24 \mathrm{~h}$ in the MRS medium supplemented with bile salts the survival rates were $96 \%$ and $100 \%$, respectively (Figure 3 ). It is important to consider that the survival rate of bacteria is usually evaluated after $3 \mathrm{~h}$; in this study, we also evaluated it at $24 \mathrm{~h}$, and found a good survival rate at pH 3.0 in bile salts. These results allow us to infer that bacteria can remain in the gastrointestinal tract despite several changes in their environmental. Lactobacillus and Enterococcus belong to the group of lactic acid bacteria; both perform similar functions, as was appreciated in this study, however when some diseases as hypercholesterolemia occurs, the Lactobacillus population decreases causing changes in the gastrointestinal environment which can be one of the main causes of the imbalance in the microbiota, although the population of Enterococcus remains constant.

\section{Acknowledgements}

This work was supported by Fondos Mixtos del Estado de Guanajuato, Grant GTO 2011-CO4-167944. Claudia Gomez wishes to thank CONACYT for scholarship No. 291204

\section{References}

1. Ley RE, Peterson DA, Gordon Jl (2006) Ecological and evolutionary forces shaping microbial diversity in the human intestine. Cell 124: 837-848.

2. Fujimura KE, Slusher NA, Cabana MD, Lynch SV (2010) Role of the gut microbiota in defining human health. Expert Rev Anti Infect Ther 8: 435-454.

3. Sekirov I, Russell SL, Antunes LC, Finlay BB (2010) Gut microbiota in health and disease. Physiol Rev 90: 859-904.

4. Marteau P, Pochart P, Doré J, Béra-Maillet C, Bernalier A, et al. (2001) Comparative study of bacterial groups within the human cecal and fecal microbiota. Appl Environ Microbiol 67: 4939-4942.

5. Lebreton F, Willems RJL, Gilmore MS (2014) Enterococcus Diversity, Origins in Nature, and Gut Colonization. (In Gilmore MS, Clewell DB, Ike Y, et al., editors) Enterococci: from commensals to leading causes of drug resistant infection. Boston Massachusetts, Eye and Ear Infirmary.

6. Klingspor S, Bondzio A, Martens H, Aschenbach JR, Bratz K, et al. (2015) Enterococcus faecium NCIMB 10415 modulates epithelial integrity, heat shock protein, and proinflammatory cytokine response in intestinal cells. Mediators Inflamm 2015: 304149

7. Roselino MN, Pauly-Silveira ND, Cavallini DC, Celiberto LS, Pinto RA, et al (2012) A potential synbiotic product improves the lipid profile of diabetic rats. Lipids Health Dis 11: 114

8. Bosch M, Fuentes MC, Audivert S, Bonachera MA, Peiró S, et al. (2014) Lactobacillus plantarum CECT 7527, 7528 and 7529: probiotic candidates to reduce cholesterol levels. J Sci Food Agric 94: 803-809.

9. Liong MT, Shah NP (2005) Bile salt deconjugation ability, bile salt hydrolase activity cholesterol co-precipitation ability of lactobacilli strains. Int Dairy J 15 391-398.

10. Lye HS, Rusul G, Liong MT (2010) Removal of cholesterol by lactobacilli via incorporation and conversion to coprostanol. J Dairy Sci 93: 1383-1392. 
Citation: Reyes-Escogido ML, Gómez-Navarro CM, Ramírez-Ardila SD, Martínez-Pérez F, Guardado-Mendoza R (2016) A Comparative Study of Some Functional Properties of Lactobacillus and Enterococcus Isolated from Feces of Normo and Hyper-Cholesterolemic Humans. $J$ Bacteriol Parasitol 7: 274. doi: 10.4172/2155-9597.1000274

11. Öner Ö, Aslim B, AydaÅŸ SB (2014) Mechanisms of cholesterol-lowering effects of lactobacilli and bifidobacteria strains as potential probiotics with their bsh gene analysis. J Mol Microbiol Biotechnol 24: 12-18.

12. Agerbaek M, Gerdes LU, Richelsen B (1995) Hypocholesterolaemic effect of a new fermented milk product in healthy middle-aged men. Eur $\mathrm{J}$ Clin Nutr 49: 346-352.

13. Hlivak P, Odraska J, Ferencik M, Ebringer L, Jahnova E, et al. (2005) One-year application of probiotic strain Enterococcus faecium M-74 decreases serum cholesterol levels. Bratisl Lek Listy 106: 67-72.

14. Vaughan CJ, Gotto AM Jr, Basson CT (2000) The evolving role of statins in the management of atherosclerosis. J Am Coll Cardiol 35: 1-10.

15. Tompkins R, Schwartzbard A, Gianos E, Fisher E, Weintraub H (2014) A current approach to statin intolerance. Clin Pharmacol Ther 96: 74-80.

16. Shi M, Zheng H, Nie B, Gong W, Cui X (2014) Statin use and risk of liver cancer: an update meta-analysis. BMJ Open 4: e005399.

17. Martinez-Gonzalez MA, Lopez-Fontana C, Varo JJ, Sanchez-Villegas A Martinez JA (2005) Validation of the spanish version of the physical activity questionnaire used in the Nurses' Health Study and the Health Professionals' Follow-up Study. Public Health Nutr 8: 920-927.

18. CLSI (2013) Performance Standards for Antimicrobial Susceptibility Testing 23th Informational Supplement Clinical and Laboratory Standards Institute document, Wayne, PA, M100-S23.

19. Bao Y, Zhang Y, Zhang Y, Liu Y, Wang S, et al. (2010) Screening of potential probiotic properties of Lactobacillus fermentum isolated from traditional dairy products. Food Control 21: 695-701.

20. Vancanneyt M, Mengaud J, Cleenwerck I, Vanhonacker K, Hoste B, et al (2004) Reclassification of Lactobacillus kefirgranum Takizawa et al. 1994 as Lactobacillus kefiranofaciens subsp. kefirgranum subsp. nov. and emended description of $L$. kefiranofaciens Fujisawa et al. 1988. Int J Syst Evol Microbiol 54: $551-556$

21. O'Sullivan O, Cronin O, Clarke SF, Murphy EF, Molloy MG, et al. (2015) Exercise and the microbiota. Gut Microbes 6: 131-136.

22. Petriz BA, Castro AP, Almeida JA, Gomes CP, Fernandes GR, et al. (2014) Exercise induction of gut microbiota modifications in obese, non-obese and hypertensive rats. BMC Genomics 15: 511.

23. Hold GL (2014) The gut microbiota, dietary extremes and exercise. Gut 63 1838-1839.
24. Gerritsen J, Smidt H, Rijkers GT, de Vos WM (2011) Intestinal microbiota in human health and disease: the impact of probiotics. Genes Nutr 6: 209-240.

25. Tehrani AB, Nezami BG, Gewirtz A, Srinivasan S (2012) Obesity and its associated disease: a role for microbiota? Neurogastroenterol Motil 24: 305-311.

26. Queipo-Ortuno MI, Seoane LM, Murri M, Pardo M, Gomez-Zumaquero JM et al. (2013) Gut microbiota composition in male rat models under different nutritional status and physical activity and its association with serum leptin and ghrelin levels. PLoS One 8: e65465.

27. Dunne C, O'Mahony L, Murphy L, Thornton G, Morrissey D, et al. (2001) In vitro selection criteria for probiotic bacteria of human origin: correlation with in vivo findings. Am J Clin Nutr 73: 386S-392S

28. Zhang T, Chen Y, Wang Z, Zhou Y, Zhang S, et al. (2013) Changes of fecal flora and its correlation with inflammatory indicators in patients with inflammatory bowel disease. Nan Fang Yi Ke Da Xue Xue Bao 33: 1474-1477.

29. Fijan S (2014) Microorganisms with claimed probiotic properties: an overview of recent literature. Int J Environ Res Public Health 11: 4745-4767.

30. Jett BD, Huycke MM, Gilmore MS (1994) Virulence of enterococci. Clin Microbiol Rev 7: 462-478.

31. Ashraf R, Shah NP (2011) Antibiotic resistance of probiotic organisms and safety of probiotic dairy products. Int Food Res J 18: 837-853.

32. Gueimonde M, Sánchez B, G de Los Reyes-Gavilán C, Margolles A (2013) Antibiotic resistance in probiotic bacteria. Front Microbiol 4: 202.

33. Torres C, Cercenado $E$ (2010) Interpretive reading of the antibiogram in gram positive cocci. Enferm Infecc Microbiol Clin 28: 541-553.

34. Liasi SA, Azmi TI, Hassan MD, Shuhaimi M, Rosfarizan M, et al. (2009) Antimicrobial activity and antibiotic sensitivity of three isolates of lactic acid bacteria from fermented fish product, Budu. Mal J Microbiol 5: 33-37.

35. Fukao M, Yajima N (2012) Assessment of antibiotic resistance in probiotic lactobacilli. In: InTechopen, editor. Antibiotic resistant bacteria - A continuous challenge in the new millennium. Rijeka, Croatia. pp: 503-512.

36. Zhou JS, Pillidge CJ, Gopal PK, Gill HS (2005) Antibiotic susceptibility profiles of new probiotic Lactobacillus and Bifidobacterium strains. Int J Food Microbio 98: 211-217

37. Bourouni OC, El Bour M, Calo-Mata P, Barros-Velazquez J (2012) Antimicrobia resistance and potential probiotic application of Enterococcus spp. in sea bass and sea bream aquaculture. In: Pana $\mathrm{M}$, editor Antibiotic resistant bacteria. A continuous challenge in the new millenium InTech. pp: 513-530. 